\title{
Comparison of Endoscopic Dysplasia Detection Techniques in Patients With Ulcerative Colitis: A Systematic Review and Network Meta-analysis
}

\author{
Talat Bessissow, MD, MSC, ${ }^{*}$ Parambir S. Dulai, MD, ${ }^{+}$Sophie Restellini, MD, ${ }^{*}$ Tara Landry, MLIS, \\ Raf Bisschops, MD, PhD, ${ }^{\S}$ Mohammad Hassan Murad, MD, MPH," and Siddharth Singh, MD, MS
}

Background: We assessed the comparative efficacy of different dysplasia detection techniques in patients with ulcerative colitis (UC) through a network meta-analysis and rated the quality of evidence using GRADE approach.

Methods: Through a systematic literature review of multiple databases through June 30, 2017, we identified parallel-group randomized controlled trials (RCTs) in adults with long-standing UC undergoing surveillance colonoscopy with standard definition-white light endoscopy (SD-WLE), high-definition WLE (HD-WLE), narrow band imaging (NBI), or dye-based chromoendoscopy. The primary outcome was the total number of dysplastic lesions. Pairwise and network meta-analysis was performed; ranking was assessed using surface under the cumulative ranking (SUCRA) probabilities.

Results: Based on 8 parallel-group RCTs (924 patients), low-quality evidence supports chromoendoscopy over SD-WLE (odds ratio [OR], 2.37; 95\% credible interval [CrI], 0.81-6.94) for any dysplasia detection, whereas very low-quality evidence supports using HD-WLE or NBI over SD-WLE (HD-WLE [vs SD-WLE]: OR, 1.21; 95\% CrI, 0.30-4.85; NBI: OR, 1.68; 95\% CrI, 0.54-5.22). Very low-quality evidence from indirect comparative analysis supports the use of chromoendoscopy over HD-WLE (OR, 1.96; 95\% CrI, 0.72-5.34) or NBI (OR, 1.41; 95\% CrI, $0.70-2.84$ ) for any dysplasia detection. The number of patients with advanced neoplasia was very small, precluding meaningful analysis.

Conclusions: Although we did not find any single technique to be superior, chromoendoscopy is probably more effective than SD-WLE for detecting any dysplasia, and there is low confidence in estimates supporting its use over HD-WLE or NBI. There is very low-quality evidence to inform the comparative efficacy of these interventions in detecting advanced neoplasia or preventing future colorectal cancer. Pragmatic, parallel-group RCTs with longitudinal follow-up are warranted to inform optimal dysplasia surveillance techniques.

Key Words: dysplasia, chromoendoscopy, narrow band imaging, white light

\section{INTRODUCTION}

Long-standing ulcerative colitis (UC) puts patients affected at an increased risk of colonic dysplasia and colorectal cancer (CRC). ${ }^{1}$ The risk of colorectal cancer has been shown in multiple studies to be higher than in the general population, although the magnitude of that risk may be decreasing. ${ }^{2}$ The cumulative incidence of CRC after 30 years of disease is estimated to be $10 \%{ }^{3}$ Due to this increased risk, guidelines recommend routine surveillance colonoscopies in patients with left-sided or extensive UC after $8-10$ years of disease, every $1-3$ years.

Received for publications January 14, 2018; Editorial Decision April 5, 2018.

From the *Division of Gastroenterology, McGill University Health Center, Montreal, Québec, Canada; Division of Gastroenterology, University of California San Diego, La Jolla, California; ${ }^{\star}$ Medical Library, McGill University Health Center, Montreal, Québec, Canada; ${ }^{\S}$ Department of Gastroenterology, University Hospitals Leuven, KU Leuven, Leuven, Belgium; "Robert D and Patricia E Kern Center for the Science of Health Care Delivery, Mayo Clinic, Rochester, Minnesota

Conflicts of interest: T.B. received honoraria as a consultant for Abbvie, Pfizer, Takeda, and Janssen; has been a speaker for Abbvie, Takeda, Shire, Janssen, Pendopharm, and Ferring; has received research funding from Abbvie, Janssen, and Pentax. S.R. has no conflicts of interest. P.S.D. received research support from Takeda and Pfizer. R.B. received honoraria as a speaker for Pentax, Fujifilm, and Norgine; has been a consultant for Pentax, Fujifilm, and Norgine; has received research funding from Pentax, Fujifilm, and Cook. T.L. has no conflicts of interest. M.H.M. has no conflicts of interest. S.S. received research grants from Pfizer and
Until recently, the gold standard for surveillance colonoscopy was to perform random 4-quadrants biopsies every $10 \mathrm{~cm}$ once the cecum was reached in addition to biopsies of any visible mucosal lesions. ${ }^{4}$ There are many limitations to this approach. First, adherence to this laborious protocol is poor. ${ }^{5-8}$ Second, the yield of random biopsies for dysplasia detection is very poor. In a Dutch study, out of 11,722 random biopsies, only $24(0.2 \%)$ were positive for dysplasia (23 low-grade dysplasia and 1 high-grade dysplasia). ${ }^{9}$ To overcome limitations of standard definition (SD-WLE) or high-definition white light

AbbVie and career development support from ACG and the Crohn's and Colitis Foundation.

Supported by: No funding was obtained to complete this work.

Author contributions: concept and design of the study (T.B., P.S.D., R.B., S.S., M.H.M.), data collection (T.B., P.S.D., S.R., T.L., S.S.), data analysis and interpretation (T.B., P.S.D., S.S., R.S., R.B., M.H.M.), drafting of the manuscript (T.B., S.R., P.S.D., M.H.M., T.L., R.B., S.S.), critical appraisal and final approval of the manuscript (T.B., P.S.D., S.R., R.B., T.L., M.H.M., S.S.).

Address correspondence to: Talat Bessissow, MD, MSc, FRCPC, Division of Gastroenterology Montreal General Hospital McGill University Health Center, 1650 Avenue Cedar Montreal, Quebec, Canada H3G 1A4 (talat.bessissow@mcgill.ca).

(C) 2018 Crohn's \& Colitis Foundation. Published by Oxford University Press. All rights reserved. For permissions, please e-mail: journals.permissions@oup.com.

doi: $10.1093 /$ ibd/izy 188 Published online 25 May 2018 
endoscopy (HD-WLE) with random biopsies, several enhanced endoscopic imaging techniques have been developed to improve dysplasia detection, including dye-based chromoendoscopy (DCE) or virtual chromoendoscopy, such as narrow-band imaging $(\mathrm{NBI}){ }^{10} \mathrm{DCE}$ has been shown to be superior to SD-WLE in cohort studies and prospective tandem studies for dysplasia detection, but data comparing DCE with HD-WLE are scant. No difference has been detected in rates of dysplasia detection between NBI and DCE, ${ }^{11}$ or NBI and HD-WLE, ${ }^{12}$ in parallel-group randomized controlled trials (RCTs).

Recent consensus statements strongly recommend DCE over WLE when using SD scopes and suggest using it over WLE when using HD scopes for dysplasia detection. In contrast, these statements suggest against using NBI over WLE or DCE, with either SD or HD scopes. However, the underlying evidence from these was derived from a mix of cohort, prospective tandem, and parallel-group RCTs and relied only on head-to-head comparisons. Prospective tandem studies invariably bias results in favor of the second modality by assuming that all lesions identified using the first modality would have been detected by the second modality. Moreover, for these consensus statements, dysplasia detection was considered a patient-relevant outcome, but the impact of these techniques on detection of advanced neoplasia or prevention of future colorectal cancer could not be inferred.

Given the lack of evidence from head-to-head trials, a network meta-analysis may be helpful in evaluating the comparative efficacy of several endoscopic interventions and synthesizing evidence across a network of RCTs. This indirect assessment of the different endoscopic techniques, adjusted by a common control, may inform patients, clinicians, and policy-makers regarding the best surveillance endoscopic technique for the detection of dysplasia in patients with ulcerative colitis. ${ }^{13}$ Therefore, we conducted a systematic review with network meta-analysis to assess the comparative efficacy of different endoscopic dysplasia detection techniques in patients with $\mathrm{UC}$, focusing only on true parallel-group RCTs. We critically appraised the quality of evidence using the GRADE ${ }^{14}$ approach using both dysplasia detection and advanced neoplasia detection as relevant outcomes.

\section{METHODS}

We performed a systematic review of an a priori established protocol and reported it according to the Preferred Reporting Items for Systematic Reviews and Meta-Analyses (PRISMA) extension statement for systematic reviews incorporating network meta-analyses for health care interventions. ${ }^{15}$ We followed good research practices delineated in the International Society for Pharmacoeconomics and Outcomes Research report on interpreting indirect treatment comparisons and network meta-analysis for health care decision-making. ${ }^{16}$

\section{Data Sources and Search Strategy}

To identify the studies that met our inclusion criteria, we systematically searched the following electronic databases from inception through June 30, 2017: MEDLINE, EMBASE, The
Cochrane Central Register of Controlled Trials, the Cochrane Database of Systematic Reviews, The Database of Abstracts of Reviews of Effects, the NHS Economic Evaluation Database, Web of Science, and Scopus. Further studies were identified in Web of Science and Scopus (June 30, 2017) by carrying out citation searches for studies citing included studies, and by inspecting their reference lists. We also searched the Clinical Trials registries to identify applicable ongoing research. Both Medical Subject Heading (MeSH) terms and free-text words were used, including the following key words: colitis, ulcerative, inflammatory bowel disease, endoscopy, colonoscopy, chromoendoscopy, narrow band imaging, colorectal neoplasia, colonic polyps, and population surveillance. No language limits were applied. The search was limited to clinical trials and human studies.

Two investigators (T.B., P.S.D.) independently evaluated the titles and abstracts of recovered studies to exclude irrelevant publications and selected relevant articles for inclusion. Disagreements were resolved through consensus and discussion with a third reviewer (S.S.).

\section{Eligibility Criteria}

We included parallel-group RCTs that met the following inclusion criteria: (1) patients: adults with long-standing UC based on endoscopic and histologic criteria (more than 8 years of disease) in clinical remission; (2) intervention: dysplasia detection using DCE, NBI, HD-WLE, or SD-WLE; (3) comparator: another enhanced imaging modality or SD-WLE; and (4) outcome: proportion of patients with any dysplastic lesion and proportion of patients with advanced neoplasia or colorectal cancer.

We excluded observational comparative studies or tandem colonoscopy studies (which assume that a second procedure would have detected all lesions identified by the first procedure, to assess incremental yield), pediatric studies (patients age $<18$ years), studies with missing data despite contacting the primary authors, and studies restricted to patients with concomitant primary sclerosing cholangitis.

\section{Outcomes}

The primary outcome of interest was the total number of dysplastic lesions. Secondary outcomes were proportion of patients with advanced neoplasia (high-grade dysplasia and/ or colorectal cancer) and rates of subsequent colorectal cancer. Any dysplasia was defined as any lesion with a pathologic diagnosis of low- or high-grade dysplasia (including tubular adenoma, tubulovillous adenoma, villous adenoma, and sessile serrated adenoma with dysplasia) or colorectal cancer.

\section{Data Extraction}

Three reviewers (T.B., S.R., P.S.D.) extracted the data independently. The following information was collected from each study: (1) study characteristics: first author's last name, year of publication, geographical location, study design and duration, and inclusion/exclusion criteria; (2) patient characteristics: age, 
sex, smoking status, personal and family history of dysplasia and colon cancer, primary sclerosing cholangitis, number of patients in each group; (3) disease characteristics: disease duration and distribution, disease activity at time of endoscopy (quiescent or mildly active disease), and medical therapy at time of endoscopy; (4) procedure characteristics: technique performed, dye used, total duration of colonoscopy, withdrawal time, bowel preparation (proportion of patients with study-defined "inadequate bowel prep"), random biopsy protocol performed or not, and total number of biopsies; (5) lesion characteristics: visible or polypoid lesions vs flat or invisible lesions, total number of dysplastic lesions (low-grade dysplasia or advanced neoplasia), and number of patients with dysplastic lesions. Risk of bias was assessed using the Cochrane risk of bias tool (Figs. 1 and 2).

\section{Data Synthesis and Analysis}

Pooled odds ratios (ORs) and 95\% confidence intervals (CIs) were calculated using the DerSimonian-Liard random-effects model. ${ }^{17}$ We assessed statistical heterogeneity using the $I^{2}$ statistic, with values greater than $50 \%$ suggesting substantial heterogeneity. ${ }^{18}$ Due to a paucity of trials, formal assessment of funnel plot symmetry was not performed. Direct comparisons were performed using RevMan, v5.3 (Cochrane Collaboration, Copenhagen, Denmark).

Next, we conducted network meta-analysis using a multivariate consistency model, random-effects meta-regression as described by Ian White, using STATA, v13.0 (College Station, TX, USA). ${ }^{19}$ This frequentist approach provides a point estimate from the network, along with $95 \%$ CIs from the frequency distribution of the estimate. In addition, sensitivity analysis was performed using random-effects Bayesian network meta-analysis, using Markov chain Monte Carlo methods in WinBUGS, v1.4.3 (MRC Biostatistics Unit, Cambridge, UK), following methods described by $\mathrm{Lu}$ and Ades. ${ }^{20}$ We modeled the comparative efficacy of any 2 techniques as a function of each technique relative to the reference technique (ie, SD-WLE in this study). This approach assumes the "consistency" of technique effects across all included trials - that is, the direct and indirect estimates are the same effects. Network consistency was evaluated by comparing the direct estimates with the indirect estimates using a node-splitting technique. We estimated the posterior distribution of all parameters using noninformative priors to limit inference to data derived from the trials at hand (ie, we made no assumptions about the effectiveness of these techniques from data published in studies that were not included in this analysis). We updated Markov chain Monte Carlo model with 50,000 simulated draws after a burn-in of 5000 iterations. The median of the posterior distribution based on 50,000 simulations was reported as the point estimate (ORs), and the corresponding 95\% credible intervals (CrIs; or Bayesian CIs) were achieved using the 2.5 th and 97.5 th percentiles of the posterior distribution, after adjusting for multiple-arm trials.

We calculated the relative ranking of techniques for dysplasia detection as their surface under the cumulative ranking (SUCRA), ${ }^{21}$ representing the percentage of efficacy attained by a technique compared with an imaginary technique that is always the best without uncertainty (ie, SUCRA $=100 \%$ ). Higher SUCRA scores signify higher ranking for dysplasia detection.

We also generated estimates of absolute event rates (or absolute risk) using the GRADEpro, v3.6 (McMaster University, 2014). The estimated risk difference (RD; better known as absolute risk reduction) was calculated by combining the odds ratio (OR) for each procedure against HD-WLE and presumed a $10 \%$ HD-WLE dysplasia detection rate as the assumed control risk $(\mathrm{ACR})$ by using the formula: risk difference $=\left(\mathrm{OR}^{*} \mathrm{ACR}-\right.$ $\mathrm{ACR}+\mathrm{ACR} * \mathrm{ACR}-\mathrm{OR} * \mathrm{ACR} * \mathrm{ACR}) /(1-\mathrm{ACR}+\mathrm{OR} * \mathrm{ACR})$. After calculating the risk difference, which is the difference between the event rates in the intervention and control groups, it was added back to the ACR to yield an approximation of the absolute risk for each procedure; $95 \%$ confidence intervals for the estimates were produced using the $95 \%$ confidence intervals of the odds ratios generated from the above calculations.

\section{Quality of Evidence (or Confidence in Estimates)}

We followed the GRADE approach to assess the confidence in estimates derived from network meta-analysis of

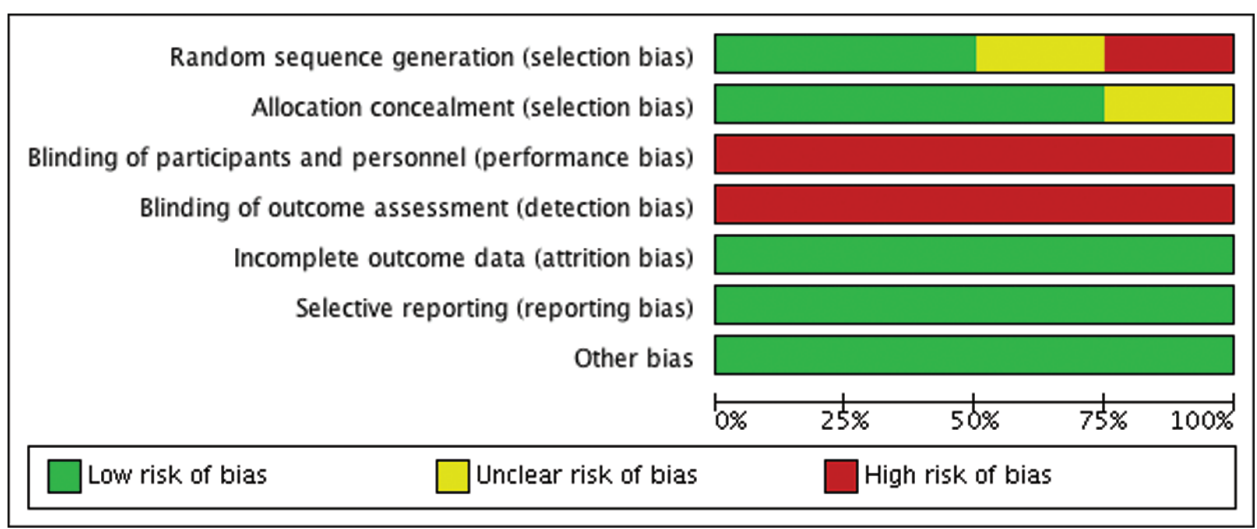

Figure 1. Cochrane risk of bias graph: review authors' judgments about each risk of bias item presented as percentages across all included studies. 


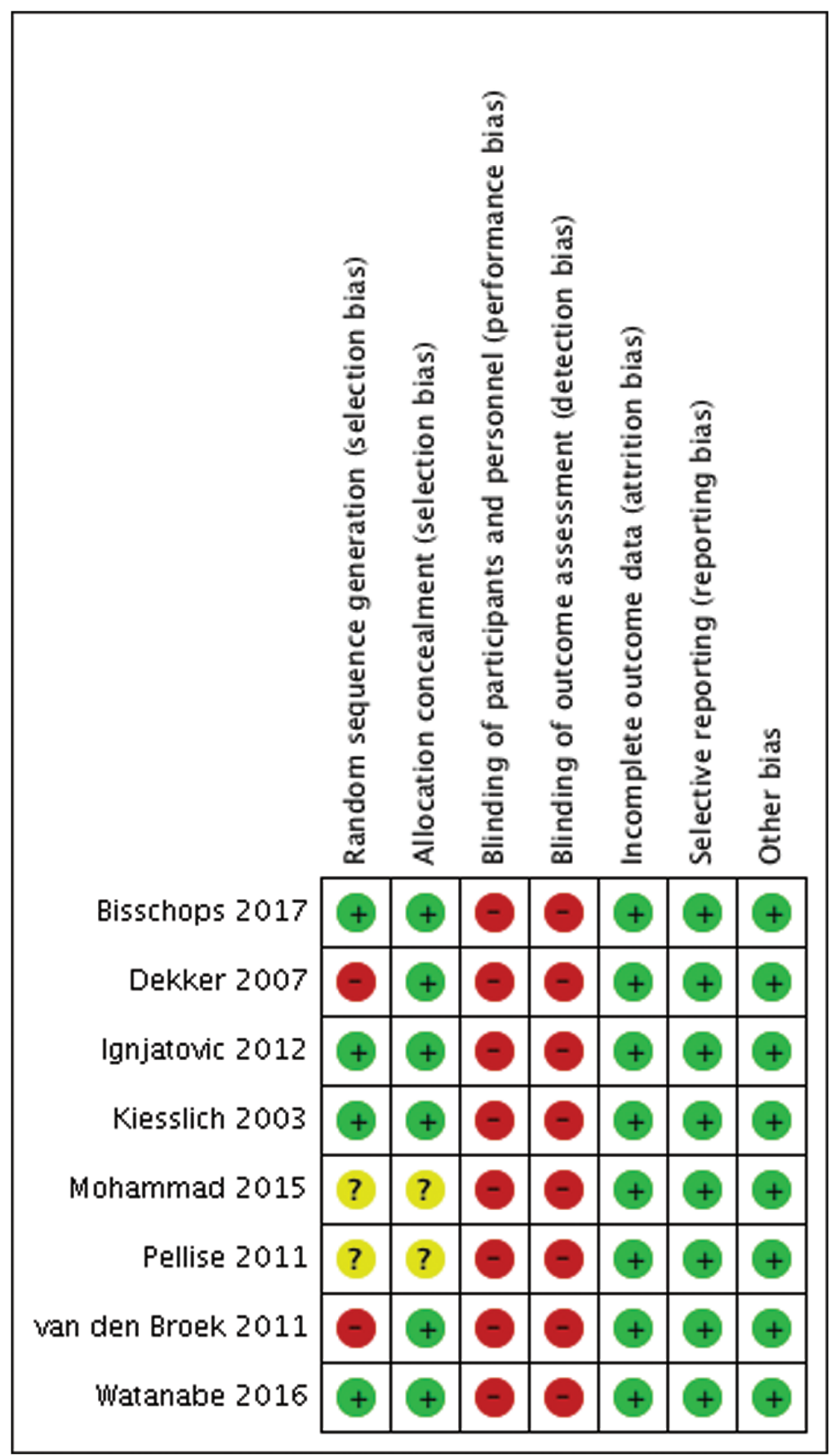

FIGURE 2. Risk of bias summary: review authors' judgments about each risk of bias item for each included study.

efficacy outcomes. ${ }^{14}$ By using this methodology, direct evidence from RCTs starts at high confidence and can be rated down based on risk of bias, indirectness, imprecision, inconsistency (or heterogeneity), and/or publication bias to levels of moderate, low, and very low confidence. The rating of indirect estimates starts at the lowest rating of the 2 pairwise estimates that contribute as first-order loops to the indirect estimate but can be rated down further for imprecision or intransitivity (dissimilarity between studies in terms of clinical or methodological characteristics). If both direct and indirect estimates were comparable (ie, coherent), then the higher of their ratings could be assigned to the network meta-analysis estimates. 


\section{RESULTS}

Our literature search identified 2353 records through database searching and citation tracking (Fig. 3). Following title, abstract, andfull-textreview when available, 8 parallel-groupRCTs were eligible, and they included a total of 924 patients. ${ }^{11,12,22-27}$
All included studies were cross-sectional studies on dysplasia detection. We did not identify any longitudinal RCT in which patients were randomized to different dysplasia detection techniques and then followed longitudinally to identify rates of development of colorectal cancer or advanced neoplasia.
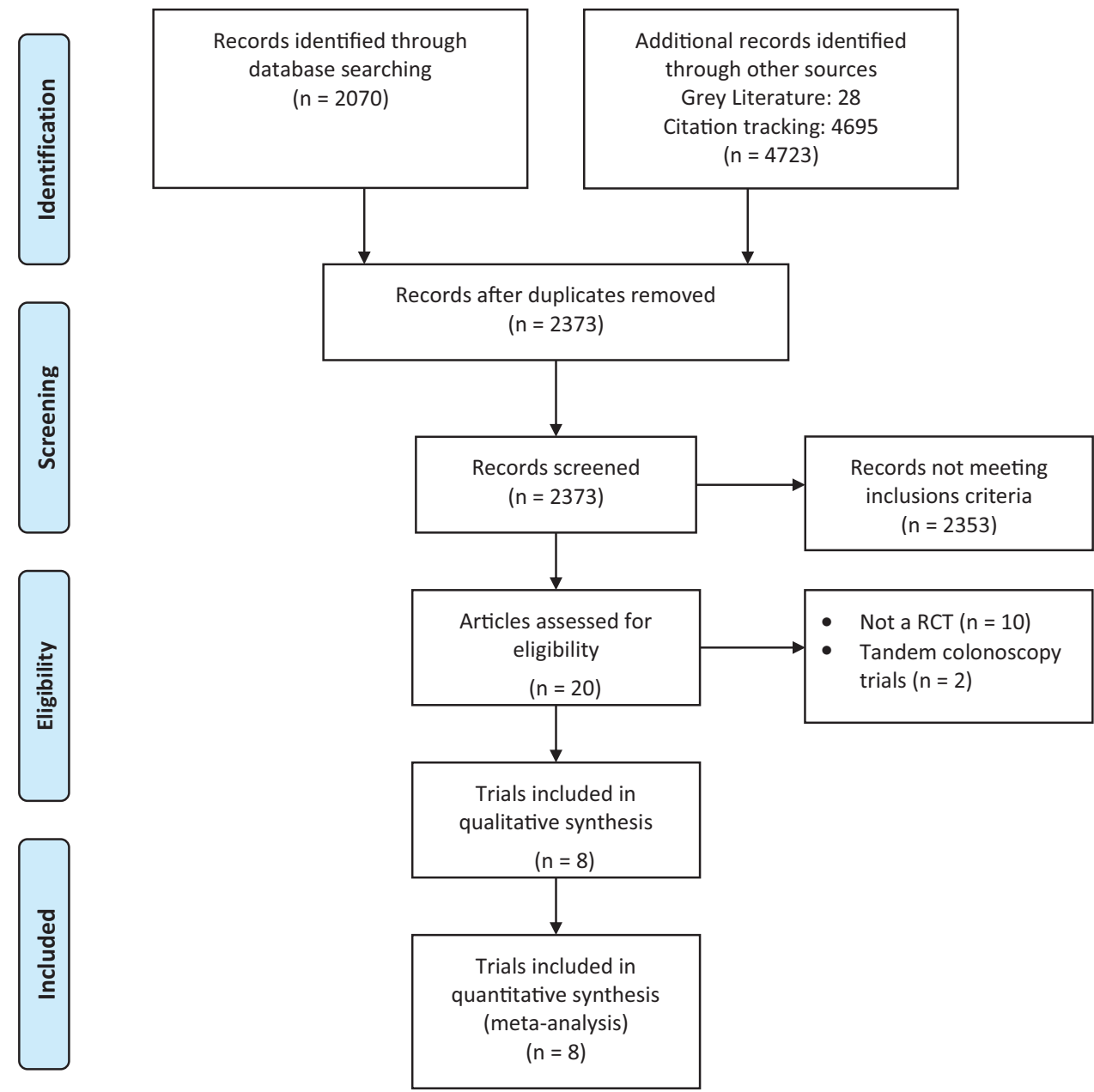

FIGURE 3. Summary of evidence search and selection.

TABLE 1: Randomized Controlled Trials Included in the Network

\begin{tabular}{|c|c|c|c|c|c|c|c|}
\hline Study Included, Date & Year & $\begin{array}{l}\text { Comparison } \\
\text { Group } 1\end{array}$ & $\begin{array}{c}\text { Comparison } \\
\text { Group } 2\end{array}$ & $\begin{array}{l}\text { Group 1, } \\
\text { No. }\end{array}$ & $\begin{array}{l}\text { Group 2, } \\
\text { No. }\end{array}$ & $\begin{array}{c}\text { Dysplasia Group 1, } \\
\text { No. }(\%)\end{array}$ & $\begin{array}{c}\text { Dysplasia } \\
\text { Group 2, No. (\%) }\end{array}$ \\
\hline Kiesslich $^{22}$ & 2003 & DCE & SD-WLE & 84 & 81 & $13(15)$ & $6(7)$ \\
\hline Dekker $^{12}$ & 2007 & NBI/SD-WLE & SD-WLE/NBI & 20 & 22 & $8(40)$ & $7(32)$ \\
\hline Pellise $^{25}$ & 2011 & DCE/NBI & NBI/DCE & 27 & 33 & $5(19)$ & $8(24)$ \\
\hline Van den Broek ${ }^{26}$ & 2011 & HD-WLE/NBI & NBI/HD-WLE & 25 & 23 & $9(36)$ & $8(35)$ \\
\hline Ignjatovic $^{23}$ & 2012 & NBI & HD-WLE & 56 & 56 & $5(9)$ & $5(9)$ \\
\hline Mohammad $^{24}$ & 2015 & DCE & HD-WLE & 50 & 53 & $11(22)$ & $5(9)$ \\
\hline Watanabe $^{27}$ & 2016 & DCE & NBI & 130 & 133 & $5(4)$ & $8(6)$ \\
\hline Bisschops $^{11}$ & 2017 & DCE & NBI & 66 & 65 & $14(21)$ & $14(22)$ \\
\hline
\end{tabular}




\section{Characteristics of Included Studies}

Chromoendoscopy was compared with SD-WLE and HD-WLE in 1 study each, whereas NBI was compared with SD-WLE in 1 study and with HD-WLE in 2 studies. Chromoendoscopy was compared with NBI in 3 studies. The number of patients included in the study ranged from 42 to 263 (Table 1). Characteristics of patients included in the study and recorded withdrawal times are presented in Supplementary Table 1. The rate of dysplasia detection ranged between $4 \%$ and $40 \%$. A total of 200 dysplastic lesions were detected, out of

TABLE 2: Neoplastic Lesions Identified With Each Endoscopic Technique

\begin{tabular}{lccccc}
\hline & Total Procedure Performed, No. & Dysplasia $(\mathrm{n}=200)$ & LGD $(\mathrm{n}=172)$ & HGD $(\mathrm{n}=22)$ & CRC $(\mathrm{n}=6)$ \\
\hline SD-WLE & 103 & 12 & 6 & 5 & 1 \\
HD-WLE & 134 & 24 & 22 & 2 & 0 \\
NBI & 330 & 58 & 51 & 4 & 3 \\
Chromoendoscopy & 357 & 106 & 93 & 11 & 2
\end{tabular}

Abbreviations: LGD, low-grade dysplasia; HGD, high-grade dysplasia.

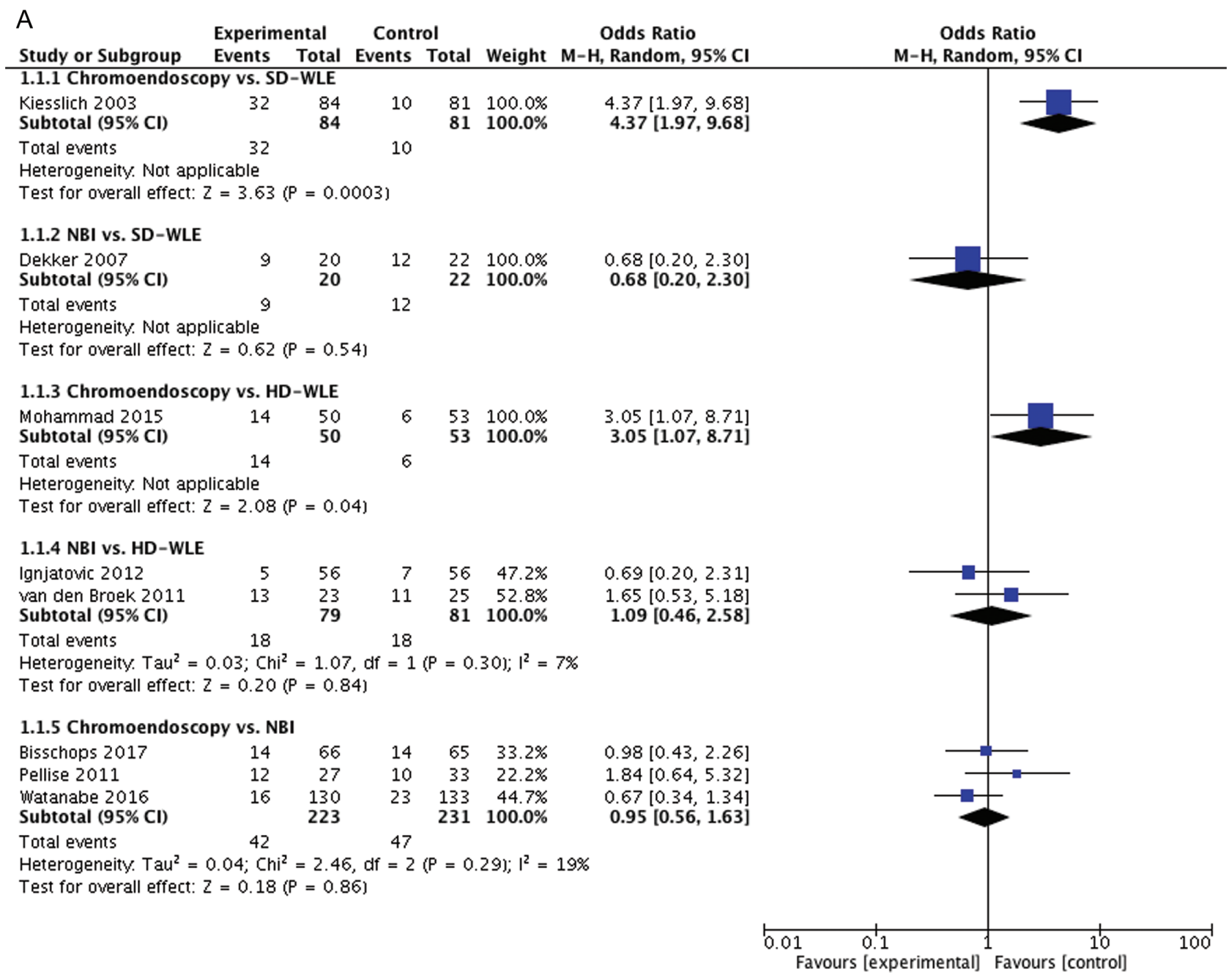

FIGURE 4. Pairwise meta-analysis comparing dysplasia detection techniques for detection of (A) any dysplastic lesion and (B) advanced neoplasia detection. 


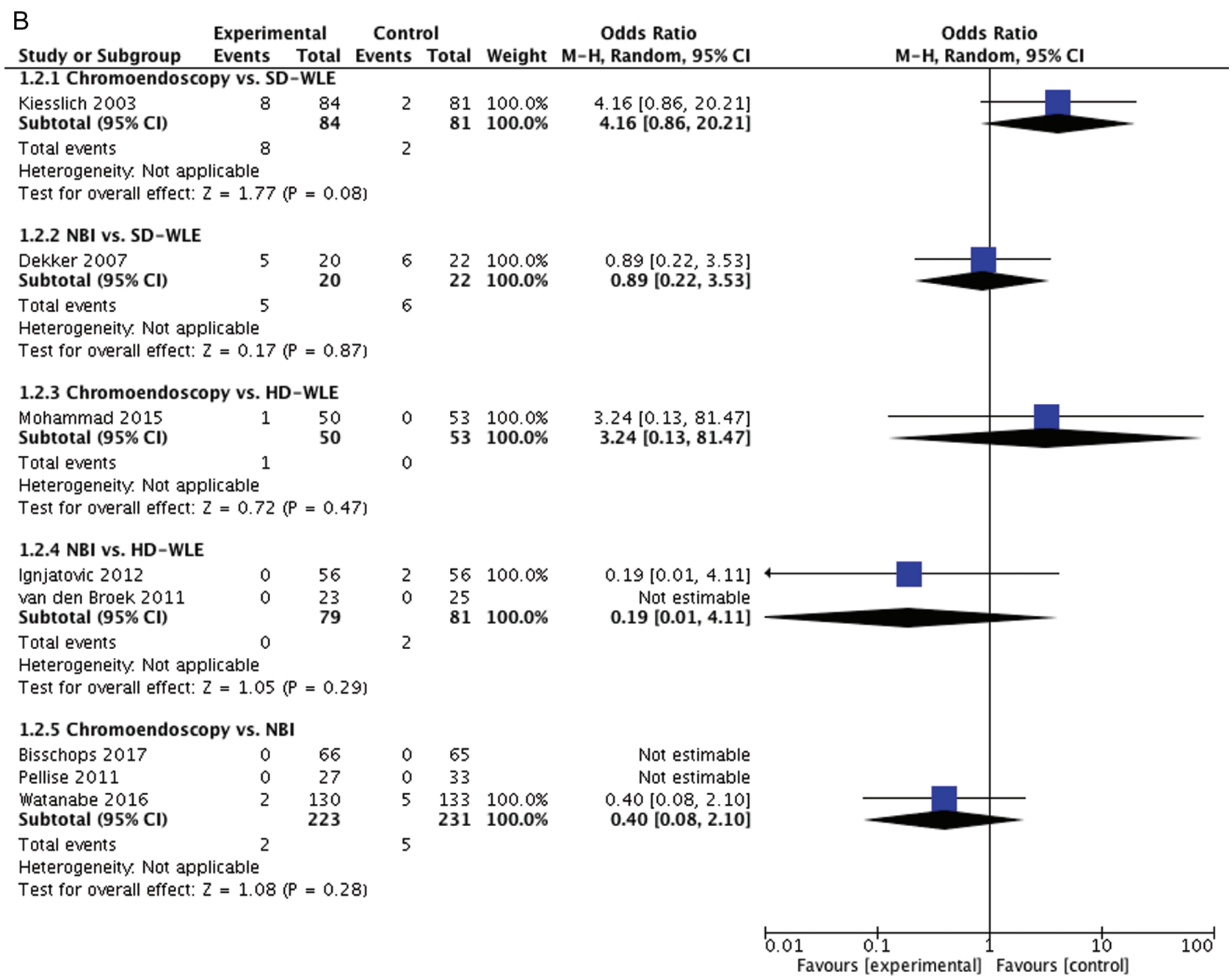

FIGURE 4. Continued

which half $(53 \%, \mathrm{n}=106$ in 357 procedures) were identified with DCE, and the remaining with NBI ( $\mathrm{n}=58$ in 330 procedures), HD-WLE ( $n=24$ in 134 procedures), and SD-WLE ( $n=12$ in 103 procedures). The majority of lesions identified were lowgrade dysplasia $(\mathrm{n}=172)$, with most of them being identified with DCE $(54 \%, \mathrm{n}=93)$. High-grade dysplasia was recognized 22 lesions, and colorectal cancer was detected in 6 lesions (Table 2). All trials were deemed to be at high risk of bias due to inadequate blinding, which may have introduced detection and performance bias.

\section{Pairwise meta-analysis}

On head-to-head comparison of different interventions, DCE was noted to be superior to SD-WLE and to HD-WLE for detection of any dysplasia, based on single studies (Fig. 4A). On meta-analysis of 3 trials, there was no difference in dysplasia detection between DCE and NBI. As noted, there were very few advanced neoplastic lesions noted in these studies. There was no significant difference in rates of advanced neoplasia detection between different techniques (Fig. 4B).

\section{Network meta-analysis}

Combining direct and indirect comparisons in a network meta-analysis (Fig. 5), we did not identify any single technique as superior to all others in dysplasia detection (Table 3). As compared with SD-WLE, both DCE and NBI were associated with $2.4 \mathrm{x}$ and $1.7 \mathrm{x}$ odds of dysplasia detection, though these differences were not statistically significant. As compared with HD-WLE, which is considered standard of care in most endoscopy units, DCE was associated with almost 2x higher odds, whereas NBI was associated with $1.4 \mathrm{x}$ higher odds of dysplasia detection, though none of these differences were statistically significant. These results were stable on sensitivity analysis using Bayesian random-effects meta-analysis (Supplementary Table 2). Inconsistency was observed in the network loop comprising NBI, DCE, and SD-WLE. Overall, DCE was ranked 
highest (SUCRA, 0.90) for dysplasia detection, followed by NBI (SUCRA, 0.57).

Assuming a HD-WLE dysplasia detection rate of $10 \%$ in clinical practice, DCE and NBI would detect dysplasia in 17.9\% and $13.4 \%$ of patients, respectively. Due to the low number of events, network meta-analysis comparing different techniques for detecting advanced neoplasia was not performed.

\section{Quality of evidence}

Overall, low-quality evidence supports DCE over SD-WLE for dysplasia detection (rated down for imprecision due to the low number of events, risk of bias), whereas very low-quality evidence supports its use over HD-WLE or NBI (very serious imprecision, risk of bias). Similarly, very low-quality evidence supports the use of NBI over HD-WLE. Importantly, when considering detection of advanced neoplasia or future development of colorectal cancer as outcomes, all evidence was further rated down for indirectness, resulting in very low-quality evidence supporting benefit of DCE over SD-WLE or HD-WLE.

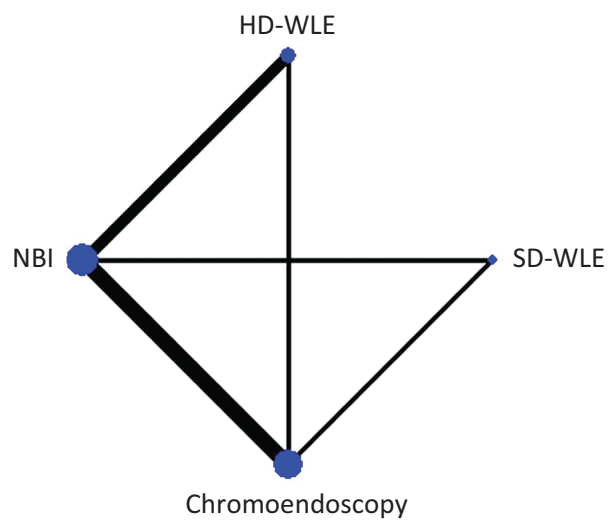

FIGURE 5. Network meta-analysis map.

\section{DISCUSSION}

Our study is the first network meta-analysis to be performed to evaluate different endoscopic techniques for the detection of dysplasia in longstanding UC. Based on 8 parallel-group RCTs, we observed that low-quality evidence supports the use of DCE over SD-WLE, whereas very low-quality evidence supports its use over HD-WLE for dysplasia detection. Due to the small number of advanced neoplastic lesions detected in trials and the paucity of longitudinal follow-up in these studies, whether use of DCE translates into decreasing colorectal cancer incidence (and consequently, mortality) in UC patients over SD-WLE or HD-WLE is uncertain. Our meta-analysis is distinct from prior studies, ${ }^{28,}{ }^{29}$ which combined observational comparative studies and prospective tandem studies with true parallel-group RCTs and, hence, likely overestimate the true benefit of DCE in dysplasia detection in UC.

Although our data support a higher dysplasia detection rate with DCE over other modalities, the majority of these lesions are low-grade dysplasia. It is unclear how higher detection of low-grade dysplasia would translate into downstream risk of developing (or preventing) colorectal cancer-whether these screen-detected low-grade dysplastic lesions represent just length-time bias without important clinical consequence remains to be seen. In a pooled analysis of 14 surveillance cohort studies in 671 patients with UC with low-grade dysplasia, the annual incidence rates of $\mathrm{CRC}$ and advanced neoplasia were $0.8 \%$ and $1.8 \%$, implying low rates of progression; these rates were much lower in studies performed with a community pathologist $(0.2 \%$ annual CRC progression) vs studies performed with an expert gastrointestinal pathologist (1.5\%). ${ }^{30} \mathrm{It}$ is conceivable, then, that with increasing detection of low-grade dysplasia in patients who undergo DCE for surveillance, especially in the community setting, a higher proportion of patients may be referred for colectomy, even though risk of progression to colorectal cancer is very low.

As with most meta-analyses, our study has important limitations. First, there was paucity of head-to-head comparisons.

TABLE 3: Network Meta-analysis Comparing Different Endoscopic Techniques for Dysplasia Detection

OR $(95 \%$ CI), Probability Intervention Superior to Comparator

\begin{tabular}{lccc} 
Comparator/Intervention & Chromoendoscopy & NBI & HD-WLE \\
\hline Chromoendoscopy & - & & SD-WLE \\
NBI & $1.41(0.70-2.84)$ & $1.39(0.54-3.54)$ & - \\
HD-WLE & $1.96(0.72-5.34)$ & $1.68(0.54-5.22)$ & $1.21(0.30-4.85)$
\end{tabular}

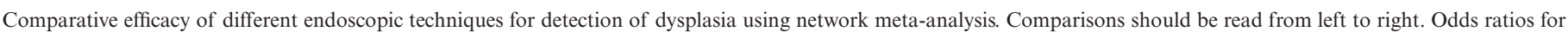

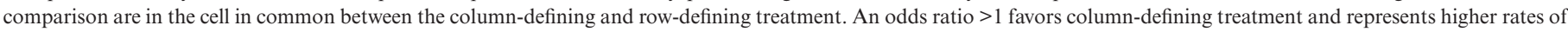
dysplasia detection. Numbers in parentheses indicate $95 \%$ confidence intervals. 
Importantly, there was a suggestion of statistical intransitivity (difference between direct and indirect comparison), though a clinical reason for the discrepancy in findings could not be determined. Second, the baseline dysplasia detection rate varied across trials, despite overall similarity in the patient population. Third, the number of studies was small, and the number of advanced neoplastic lesions was very low, which precluded meaningful analysis of this outcome.

\section{CONCLUSIONS}

Based on our network meta-analysis and applying the GRADE approach, only low-quality evidence supports the use of chromoendoscopy over SD-WLE, and very low-quality evidence supports its use over HD-WLE or NBI for dysplasia surveillance in UC. Natural history studies of screen-detected low-grade dysplasia are limited and overall suggest low rates of progression to advanced neoplasia. Hence, although DCE seems to be a promising technique for dysplasia detection, routine use in all patients may not be necessary until longer studies assessing true patient-relevant clinical impact are available.

\section{SUPPLEMENTARY DATA}

Supplementary data are available at Inflammatory Bowel Diseases online.

\section{REFERENCES}

1. Eaden JA, Abrams KR, Mayberry JF. The risk of colorectal cancer in ulcerative colitis: a meta-analysis. Gut. 2001;48:526-535.

2. Jess T, Simonsen J, Jørgensen KT, et al. Decreasing risk of colorectal cancer in patients with inflammatory bowel disease over 30 years. Gastroenterology. 2012:143:375-381 e1; quiz e13.

3. Choi CH, Rutter MD, Askari A, et al. Forty-year analysis of colonoscopic surveillance program for neoplasia in ulcerative colitis: an updated overview. Am J Gastroenterol. 2015;110:1022-1034.

4. Rubin CE, Haggitt RC, Burmer GC, et al. DNA aneuploidy in colonic biopsies predicts future development of dysplasia in ulcerative colitis. Gastroenterology. 1992; 103:1611-1620.

5. Eaden JA, Ward BA, Mayberry JF. How gastroenterologists screen for colonic cancer in ulcerative colitis: an analysis of performance. Gastrointest Endosc. 2000;51:123-128

6. Gearry RB, Wakeman CJ, Barclay ML, et al. Surveillance for dysplasia in patients with inflammatory bowel disease: a national survey of colonoscopic practice in New Zealand. Dis Colon Rectum. 2004;47:314-322.

7. Kaltz B, Bokemeyer B, Hoffmann J, et al. Surveillance colonoscopy in ulcerative colitis patients in Germany. Z Gastroenterol. 2007;45:325-331.

8. Obrador A, Ginard D, Barranco L. Review article: colorectal cancer surveillance in ulcerative colitis - what should we be doing? Aliment Pharmacol Ther. 2006;24(Suppl 3):56-63.

9. van den Broek FJ, Stokkers PC, Reitsma JB, et al. Random biopsies taken during colonoscopic surveillance of patients with longstanding ulcerative colitis: low yield and absence of clinical consequences. Am J Gastroenterol. 2014;109:715-722

10. Bessissow T, Bisschops R. Advanced endoscopic imaging for dysplasia surveillance in ulcerative colitis. Expert Rev Gastroenterol Hepatol. 2013;7:57-67.

11. Bisschops R, Bessissow T, Joseph JA, et al. Chromoendoscopy versus narrow band imaging in UC: a prospective randomised controlled trial. Gut. In press.

12. Dekker E, van den Broek FJ, Reitsma JB, et al. Narrow-band imaging compared with conventional colonoscopy for the detection of dysplasia in patients with longstanding ulcerative colitis. Endoscopy. 2007:39:216-221.

13. Cipriani A, Higgins JP, Geddes JR, et al. Conceptual and technical challenges in network meta-analysis. Ann Intern Med. 2013;159:130-137.

14. Puhan MA, Schünemann HJ, Murad MH, et al; GRADE Working Group. A GRADE working group approach for rating the quality of treatment effect estimates from network meta-analysis. BMJ. 2014;349:g5630.

15. Hutton B, Salanti G, Caldwell DM, et al. The PRISMA extension statement for reporting of systematic reviews incorporating network meta-analyses of health care interventions: checklist and explanations. Ann Intern Med. 2015;162:777-784

16. Jansen JP, Fleurence R, Devine B, et al. Interpreting indirect treatment comparisons and network meta-analysis for health-care decision making: report of the ISPOR task force on indirect treatment comparisons good research practices: part 1. Value Health. 2011;14:417-428.

17. DerSimonian R, Laird N. Meta-analysis in clinical trials. Control Clin Trials. 1986; 7:177-188.

18. Higgins JP, Thompson SG, Deeks JJ, et al. Measuring inconsistency in meta-analyses. BMJ. 2003;327:557-560

19. White IR, Barrett JK, Jackson D, et al. Consistency and inconsistency in network meta-analysis: model estimation using multivariate meta-regression. Res Synth Methods. 2012;3:111-125

20. Lu G, Ades AE. Combination of direct and indirect evidence in mixed treatmen comparisons. Stat Med. 2004;23:3105-3124.

21. Salanti G, Ades AE, Ioannidis JP. Graphical methods and numerical summarie for presenting results from multiple-treatment meta-analysis: an overview and tutorial. J Clin Epidemiol. 2011;64:163-171.

22. Kiesslich R, Fritsch J, Holtmann M, et al. Methylene blue-aided chromoendoscopy for the detection of intraepithelial neoplasia and colon cancer in ulcerative colitis. Gastroenterology. 2003;124:880-888.

23. Ignjatovic A, East JE, Subramanian V, et al. Narrow band imaging for detection of dysplasia in colitis: a randomized controlled trial. Am J Gastroenterol. 2012;107:885-890.

24. Mohammed N, Kant P, Abid F, et al. High definition white light endoscopy (HDWLE) versus high definition with chromoendoscopy (HDCE) in the detection of dysplasia in long standing ulcerative colitis: a randomised controlled trial Gut. 2015;64:A14-A15.

25. Pellisé M, López-Cerón M, Rodríguez de Miguel C, et al. Narrow-band imaging as an alternative to chromoendoscopy for the detection of dysplasia in long-standing inflammatory bowel disease: a prospective, randomized, crossove study. Gastrointest Endosc. 2011;74:840-848.

26. van den Broek FJ, Fockens P, van Eeden S, et al. Narrow-band imaging versu high-definition endoscopy for the diagnosis of neoplasia in ulcerative colitis Endoscopy. 2011;43:108-115.

27. Watanabe K, Nishishita M, Shimamoto F, et al. Comparison between newly-developed NBI and panchromoendoscopy for surveillance colonoscopy in patients with longstanding ulcerative colitis: a prospective multicenter randomized controlled trial, navigator study. J Gastroenterol Hepatol. 2016;31:138-139.

28. Har-Noy O, Katz L, Avni T, et al. Chromoendoscopy, narrow-band imaging o white light endoscopy for neoplasia detection in inflammatory bowel diseases. Dig Dis Sci. 2017:62:2982-2990.

29. Subramanian V, Mannath J, Ragunath K, et al. Meta-analysis: the diagnostic yield of chromoendoscopy for detecting dysplasia in patients with colonic inflammatory bowel disease. Aliment Pharmacol Ther. 2011;33:304-312.

30. Fumery M, Dulai PS, Gupta S, et al. Incidence, risk factors, and outcomes of colorectal cancer in patients with ulcerative colitis with low-grade dysplasia: a systematic review and meta-analysis. Clin Gastroenterol Hepatol. 2017;15:665-674.e5. 